\title{
74 \\ The Effect of Radiotherapy on Abnormal Eye Movements in Dysthyroid Orbitopathy
}

\author{
J. S. Elston and P. N. Plowman
}

As a result of improvements in beam collimation and positioning, radiotherapy has recently been re-introduced in the management of benign orbital diseases. We used the technique in 6 cases of dysthyroid orbitopathy with restrictive strabismus; sequential orthoptic measurements and Hess charts show a rapid response, with improvement continuing over a 2-4 month period and subsequently being maintained. Other orbital signs also improved and there was no evidence of side effects. In 3 cases a small area of binocular single vision before treatment substantially enlarged whilst in three others the surgical treatment of the strabusmus was considerably simplified. We recommend this simple, safe and effective treatment which reduces the need for orbital decompression and strabismus surgery. 